\title{
Simultaneous Structural and Electrical Analysis of Vanadium Dioxide Using In Situ TEM
}

Hessam Ghassemi ${ }^{1}$, Ben Jacobs ${ }^{1}$, Hasti Asayesh-Ardakani ${ }^{2}$, Wentao Yao ${ }^{2}$, Lucille A. Giannuzzi ${ }^{3}$, Reza Shahbazian-Yassar ${ }^{2}$

1. Protochips Inc., 3800 Gateway Centre Blvd, Suite 306, Morrisville, NC 27560, U.S.A.

2. Department of Mechanical and Industrial Engineering, University of Illinois at Chicago, Chicago, IL 60607, U.S.A.

3. EXpressLO LLC, Lehigh Acres, FL 33971, USA

Vanadium dioxide $\left(\mathrm{VO}_{2}\right)$, a correlated electron material, has received significant attentions due to its metal-insulator transition (MIT) at $\sim 67{ }^{\circ} \mathrm{C}$ [1]. This transition is associated with structural phase transition from the monoclinic (M1), an insulating phase, to rutile (R), a metallic phase. This metalinsulator transision is accompanied by a noticeable resistivity, optical transparency and magnetic changes. These distinctive properties have inspired many applications such as thermo/electrochromics, Mott transistors, memristors, thermal actuators, gas sensors, strain sensors and temperature sensors. Recent efforts focus on controlling of phase transition and domain structures in finite size $\mathrm{VO}_{2}$, which results in different material properties and play a critical role in device applications.

We investigated the $\mathrm{M} \rightarrow \mathrm{R}$ phase transition of individual $\mathrm{VO}_{2}$ nanowires (NWs) using in situ TEM. We first manipulated individual NWs between two electrical electrodes using an ex situ lift out tool [2] as shown in Figure 1a. We then heated at $5{ }^{\circ} \mathrm{C} / \mathrm{s}$ from room temperature (RT) to $130{ }^{\circ} \mathrm{C}$, and measured the electrical behavior simultaneously to correlate the evolution of resistance during the phase transition. Figure $1 \mathrm{~b}$ shows three distinct regimes of resistance change collected during the simultaneous heating and electrical in situ TEM experiments. In the first regime, heating from RT to $50{ }^{\circ} \mathrm{C}$, the $\mathrm{NW}$ transitioned to a combination of M1 and M2 phases, and the resistance drops significantly from $\sim 50 \mathrm{k}$ Ohm to $\sim 14 \mathrm{k} \mathrm{Ohm}$. In the second regime, heating from $50{ }^{\circ} \mathrm{C}$ to $100{ }^{\circ} \mathrm{C}$, the insulator phase transitioned mostly to the metal phase, and the resistance changed from $\sim 14 \mathrm{k}$ Ohm to $\sim 4 \mathrm{k}$ Ohm. In the final regime, the NW completely transitioned to the metal phase.

In situ diffraction patterns (DP) and dark field TEM images confirmed the phase transition from monoclinic (101) to tetragonal (101). Figures 2a-c represent evolution of DP of the same region shown in Figures 2d-f while the sample was heated from RT to $60{ }^{\circ} \mathrm{C}$ and $100{ }^{\circ} \mathrm{C}$. The red dotted line indicates the reaction front motion through the $\mathrm{NW}$ as the $\mathrm{M} \rightarrow \mathrm{R}$ transition occurs. We further investigated types and distributions of different intermediate phases during the $M \rightarrow R$ using weak-beam dark-field technique. Additionally, motion of domain walls and lattice constant measurements were further investigated via high-resolution in situ TEM imaging. We successfully observed, indexed and characterized the initial, intermediate and final phases of the $\mathrm{VO}_{2}$ transition, and correlated the phases to the electrical properties. These results will help lead to future development and application of phase change materials in various fields of electronics and nanodevices. 


\section{References:}

[1] V. Eyert, Ann Phys (Berlin) 11 (2002), p. 650.

[2] L. A. Giannuzzi et al., Microsc Microanal 21 (2015), p. 1034
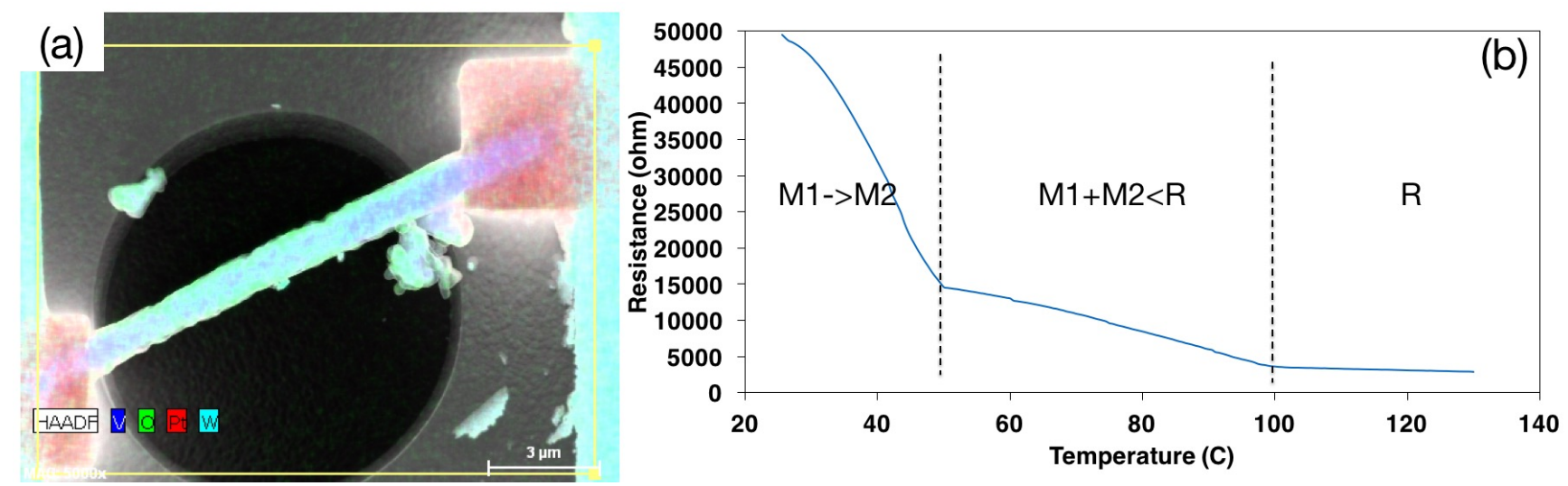

Figure 1. (a) EDS map of an individual $\mathrm{VO}_{2} \mathrm{NW}$ connected with Pt pads to $\mathrm{W}$ electrical electrodes of a MEMS E-chip device. (b) Plot of evolution of resistance as a function of temperature where clear distinction between regimes of phase transition is visible.
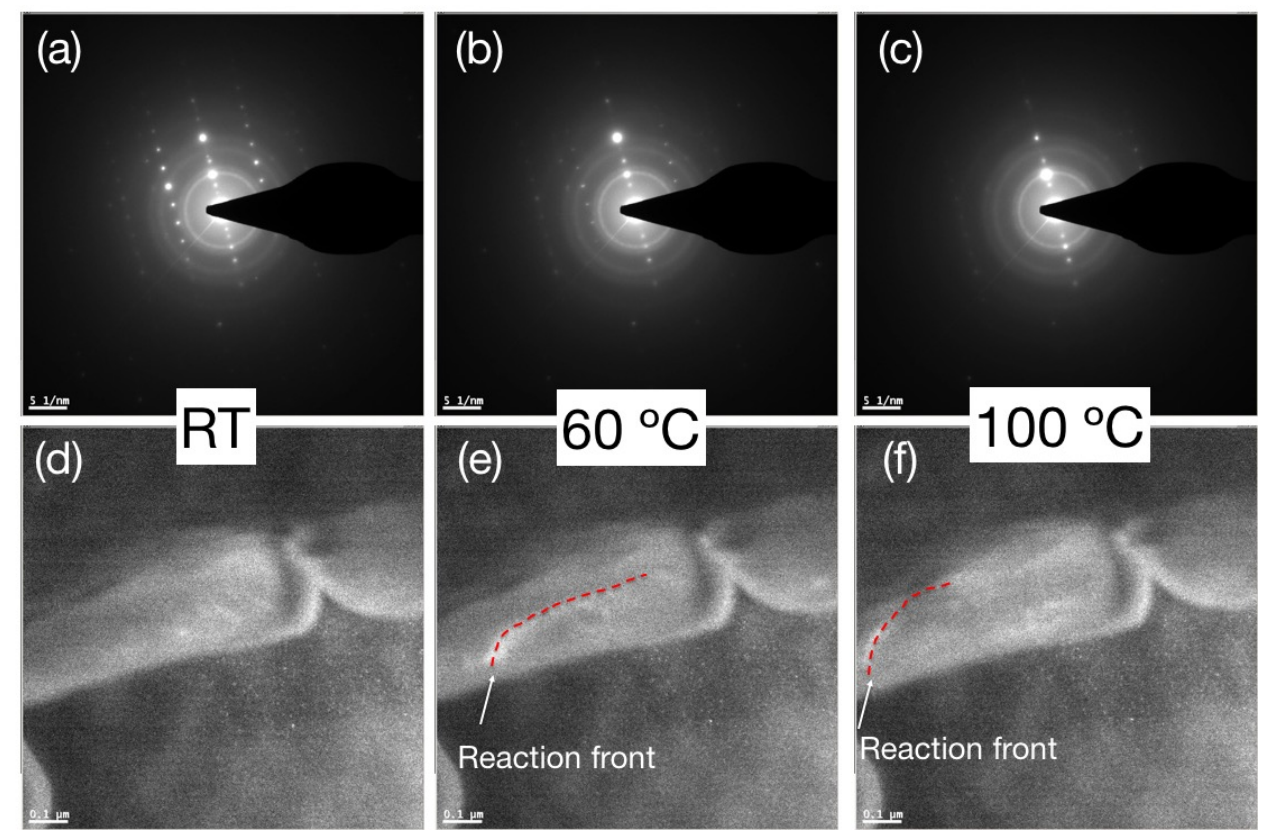

Figure 2. Snapshots of $\mathrm{M} \rightarrow \mathrm{R}$ transition. (a-c) Diffraction patterns at $\mathrm{RT}, 60^{\circ} \mathrm{C}$ and $100{ }^{\circ} \mathrm{C}$ taken from the same region as shown in dark-field images (d-f). Dark-field images illustrate reaction front motion as indicated with the red dotted line. 